\title{
Preparation of hollow hydroxyapatite microspheres utilizing poly(divinylbenzene) as a template
}

\author{
Takahiro KAWAI, ${ }^{\dagger}$ Hironobu SEKIKAWA and Hidero UNUMA \\ Graduate School of Science and Engineering, Yamagata University, 4-3-16, Jonan, Yonezawa, Yamagata $992-8510$
}

\begin{abstract}
Hollow hydroxyapatite (HAp) microspheres a few tens micrometers in diameter were fabricated by precipitating HAp preferentially on the surface of an organic polymer template using enzymatically supplied precipitant. The template, polydivinylbenzene (PDVB) microsphere encapsulating urease, was synthesized in W/O/W emulsion. HAp was precipitated on the surface of the template as ammonia was enzymatically supplied from inside of the template in a solution containing calcium ion, phosphate ion and urea. Within $21.6 \mathrm{ks}$, the surface of the template was covered with HAp. After the template was removed by calcination at $873 \mathrm{~K}$, the HAp hollow microspheres were obtained. The size of the HAp microspheres reflected that of the template and the latter was easily controlled in a range from 10 to $200 \mu \mathrm{m}$ by the preparation condition of the W/O/W emulsion. The shell thickness of the HAp sphere was also regulated by the duration of the enzymatic reaction. This material can be a promising candidate for the carrier of the drug delivery system.
\end{abstract}

(c2009 The Ceramic Society of Japan. All rights reserved.

Key-words : Hydroxyapatite, Hollow microsphere, Polymer template, Enzymatic reaction

[Received December 1, 2008; Accepted February 19, 2009]

\section{Introduction}

The "drug delivery system" is a strategy to carry a pertinent minimal amount of drugs to the aimed part of a body using "carrier" particles. So far, a number of materials have been considered to be served as the carrier, such as poly(L-lactic acid) (PLLA), ${ }^{1)}$ bioactive glass-PLLA-poly(methyl methacrylate) (PMMA) composite, ${ }^{2)}$ or aluminosilicate pellet. ${ }^{3)}$ Among them, hydroxyapatite (HAp) microsphere ${ }^{4)-11}$ is one of the potential materials for such applications due to its high bio-affinity and controllability of dissolution rate depending on surrounded environment. ${ }^{12)}$ On the other hand, since it is very important to control the specific gravity of HAp to prevent it from moving into unintended spaces, previous studies ${ }^{13)-17)}$ have shown strategies for fabrication of hollow HAp microspheres, but their sizes were either lager than $100 \mu \mathrm{m}^{13)-16)}$ or smaller than $1 \mu \mathrm{m} .{ }^{17)}$ So far, hollow HAp microspheres a few tens micrometers in diameter capable to get stuck in capillary blood vessel, have not been prepared. If HAp microspheres of this size become readily available, a new remedy of diseases might be promoted.

In the present study, we tried to fabricate hollow HAp microspheres a few tens micrometers in diameter. Our strategy involves the use of a urease-containing polymer template onto which HAp is precipitated as enzymatic hydrolysis of urea proceeds. Factors affecting the size and the shell thickness were investigated.

\section{Materials and methods}

2.1 Preparation of urease-containing PDVB template

One hundred milligrams of commercial urease powder (NAGAPSIN $^{\circledR}$, Nagase \& Co., Ltd.), $0.23 \mathrm{~g}$ of ammonium persulfate (Wako Pure Chemical Industries Ltd.) and $0.10 \mathrm{~g}$ of

\footnotetext{
Corresponding author: T. Kawai; E-mail: t-kawai@yz.yamagata-u. ac.jp
}

$N, N, N^{\prime}, N$ '-tetrametyl ethylenediamine (Wako Pure Chemical Industries Ltd., Japan) were dissolved in $5 \mathrm{~cm}^{3}$ of ion exchanged water. This solution was added into $2 \mathrm{~cm}^{3}$ of hexane where 2.7 $\mathrm{g}$ of divinylbenzene (DVB, Merck Schuchardt OHG, Germany) and $0.25 \mathrm{~g}$ of Span80 (a kind of surfactants, Kanto Chemical Co., Inc.) had been dissolved, and vigorously stirred at 10,000 rpm for $300 \mathrm{~s}$, to prepare W/O emulsion. Then this W/O emulsion was added into water containing 1 to 3 mass $\%$ of polyvinyl alcohol (PVA, Kanto Chemical Co., Inc.) as a dispersant and stirred at $300 \mathrm{rpm}$ to prepare $\mathrm{W} / \mathrm{O} / \mathrm{W}$ emulsion. The $\mathrm{W} / \mathrm{O} / \mathrm{W}$ emulsion was kept stirred at $310 \mathrm{~K}$ for $21.6 \mathrm{ks}$ in a flow of Ar to promote polymerization of DVB.

\subsection{Precipitation of HAp on the templates}

After the polymerization, $708 \mathrm{mg}$ of reagent grade $\mathrm{Ca}\left(\mathrm{NO}_{3}\right)_{2} \cdot 4 \mathrm{H}_{2} \mathrm{O}, 207 \mathrm{mg}$ of $\mathrm{NH}_{4} \mathrm{H}_{2} \mathrm{PO}_{4}$, and $1.98 \mathrm{~g}$ of urea (all the three reagents were purchased from Kanto Chemical Co., Inc.) were added to the outer water phase. The resultant concentrations of calcium, phosphate ions and urea were 10, 6, and 110 $\mathrm{mM}$, respectively. The $\mathrm{pH}$ of the solution was adjusted to 5.8 by adding an adequate amount of aqueous ammonia just before feeding urea. This solution was stirred at $200 \mathrm{rpm}$ for 21.6 to 432 ks. During this process, enzymatic hydrolysis of urea (Eq. (1)) and precipitation of HAp took place (Eq. (2)).

$$
\begin{aligned}
& \mathrm{NH}_{2} \mathrm{CONH}_{2}+3 \mathrm{H}_{2} \mathrm{O} \longrightarrow 2 \mathrm{NH}_{4}^{+}+2 \mathrm{OH}^{-}+\mathrm{CO}_{2} \\
& 10 \mathrm{Ca}^{2+}+6 \mathrm{PO}_{4}{ }^{3-}+2 \mathrm{OH}^{-} \longrightarrow \mathrm{Ca}_{10}\left(\mathrm{PO}_{4}\right)_{6}(\mathrm{OH})_{2}
\end{aligned}
$$

After predetermined time of HAp precipitation, the samples were collected by decantation, then carefully rinsed with ion exchange water and iso-propanol, and dried in air at room temperature. Then the samples were subjected to heat treatment in a muffle furnace (Taiyo, Isuzu Seisakusho Co., Ltd.) at $823 \mathrm{~K}$ for $10.8 \mathrm{ks}$ to remove the template. 


\subsection{Characterization of the samples obtained}

The morphology of the PDVB templates was observed with an optical microscope (BX51, Olympus, Co., Ltd.). Samples after HAp precipitation was characterized by thermogravimetrydifferential thermal analysis (TG-DTA 2000, Bruker AXS K. K.), X-ray diffraction (XRD, MiniFlex, Rigaku Co.), Fourier transform infra-red spectroscopy (FT-IR, FT-720, Horiba, Ltd.), and scanning electron microscopy (SEM, e-SEM, Shimadzu Rika Corp.).

\section{Results and discussion}

Figure 1 shows optical micrographs of the PDVB templates prepared under different stirring rate and PVA concentration. The size of the template ranged from 10 to $200 \mu \mathrm{m}$, and it decreased with increasing either PVA concentration or the stirring rate. Further experiments were performed using the templates $10-50 \mu \mathrm{m}$ in diameter.

Figure 2 shows TG-DTA curves of a sample after $432 \mathrm{ks}$ of precipitation. Two distinct exothermic peaks around $610 \mathrm{~K}$ and $720 \mathrm{~K}$ were observed for the DTA curve. The weight of the sample gradually decreased with increasing temperature up to $520 \mathrm{~K}$ and drastically decreased with a further increase in temperature up to $805 \mathrm{~K}$. It finally reached to a plateau over $820 \mathrm{~K}$ without any major change for the DTA curve. It is supposed that the first and second largest peaks for the DTA curve are attributed to combustion of PVA and PDVB, respectively. It is clear that the temperature enough to remove polymer components in the sample is higher than $820 \mathrm{~K}$. We chose $873 \mathrm{~K}$ (kept for 10.8 $\mathrm{ks})$ as the temperature of the heat treatment for the following experiments.

Figure 3 shows SEM images of the samples after $432 \mathrm{ks}$ of the precipitation before and after the heat treatment. The samples
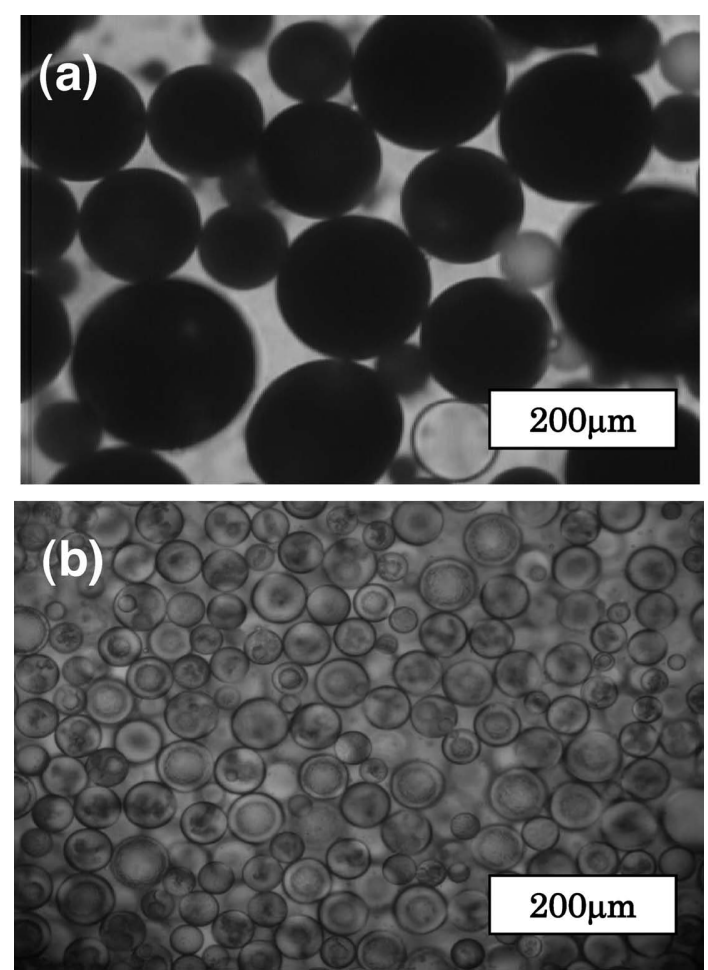

Fig. 1. Optical micrographs of the PDVB templates which underwent stirring during its polymerization in solutions with (a) 1 and (b) 3 mass $\%$ of PVA.

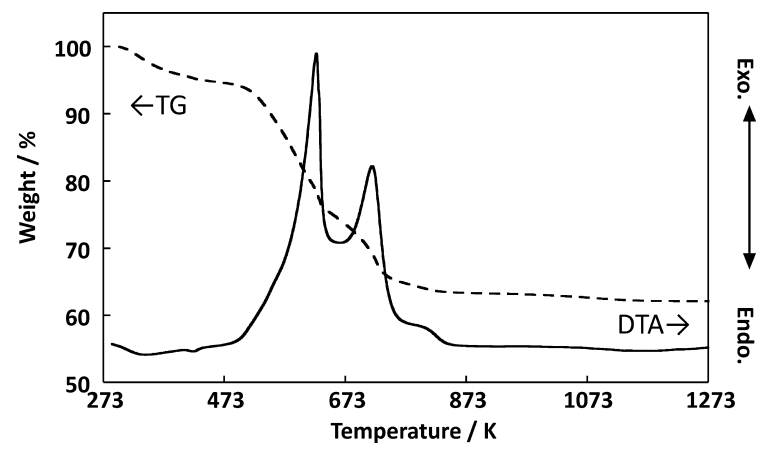

Fig. 2. TG-DTA curve of the sample after $432 \mathrm{ks}$ of the precipitation.
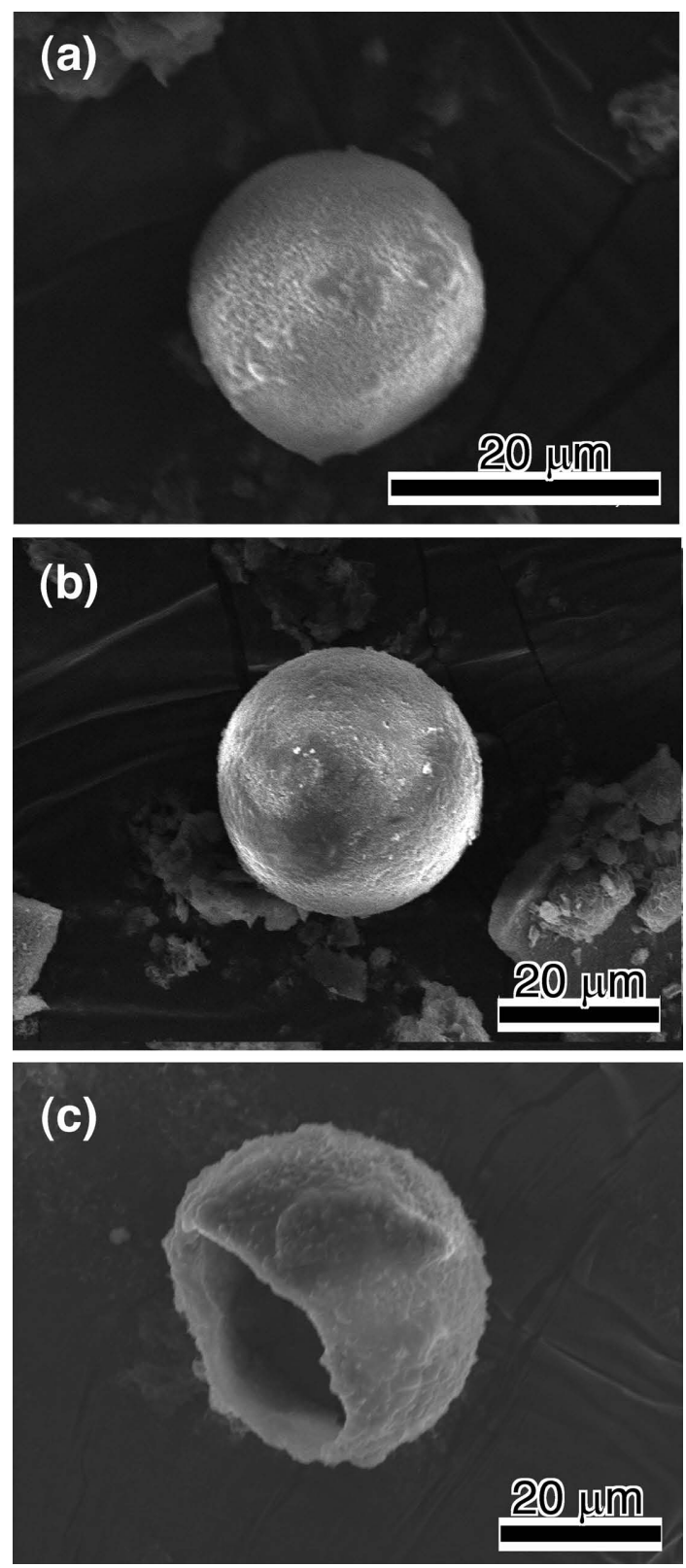

Fig. 3. SEM images of the samples after $432 \mathrm{ks}$ of the precipitation (a) before and (b,c) after the heat treatment. An accidentally broken sphere (c) shows the hollowness inside the particles. 


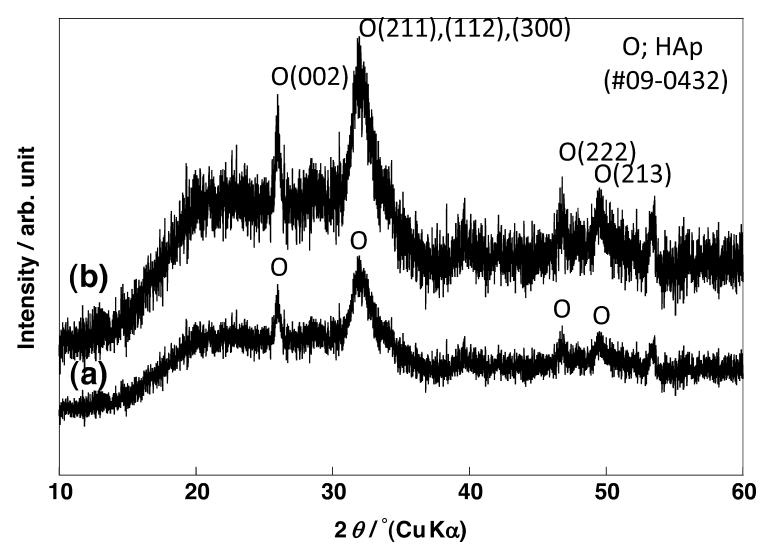

Fig. 4. XRD patterns of the samples after $432 \mathrm{ks}$ of the precipitation (a) before and (b) after the heat treatment.

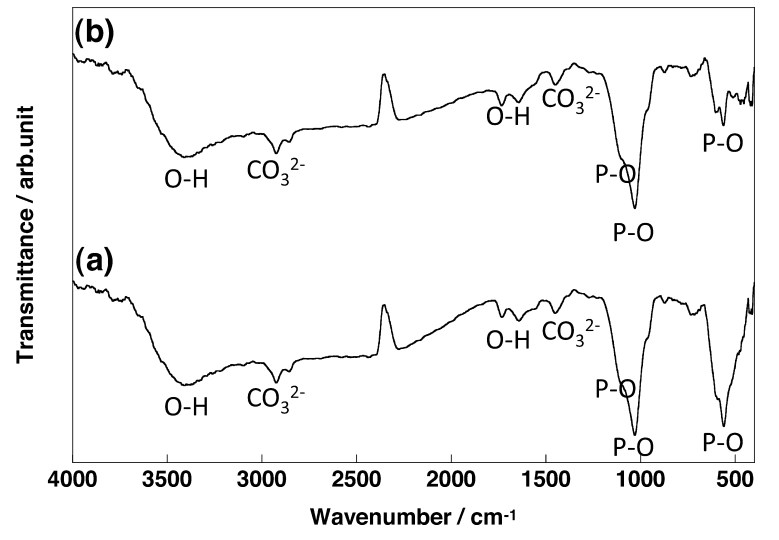

Fig. 5. FT-IR spectra of the samples after $432 \mathrm{ks}$ of HAp precipitation (a) before and (b) after the heat treatment.

both before and after heating (Figs. 3(a), (b)) were found to have spherical shape corresponding to that of the PDVB template. The particle sizes both before and after heating were ranging from 10 to $50 \mu \mathrm{m}$ in diameter, reflecting the size of PDVB template particles. The microspheres consisted of submicron-sized crystals both before and after the heat treatment. In addition, the sample microsphere after heating was found to be hollow (Fig. 3(c)). The frequency of the broken particles per all ones was almost less than $1 \%$.

Figure 4 shows XRD patterns of the samples after $432 \mathrm{ks}$ of the precipitation before and after the heat treatment. A peak at $2 \theta=26^{\circ}$ and a hallow around $32^{\circ}$ were detected for both samples before and after heating. This indicates that the precipitate was hydroxyapatite (JCPDS Card \#09-0432). As compared with a commercial calcined HAp, these samples had low crystallinity and/or small crystallite size. In addition, it is interesting to note that the crystallinity did not increase even after a heat treatment at $873 \mathrm{~K}$ for $10.8 \mathrm{ks}$. However, no apparent reason is seen why the crystallinity of even the calcined HAp was low.

Figure 5 shows FT-IR spectra of the samples after 432 ks of HAp precipitation before and after the heat treatment. Peaks at $2800 \mathrm{~cm}^{-1}$ and $1450 \mathrm{~cm}^{-1}$ corresponding to absorbance of carbonate ions are detected as well as the bands attributed to $\mathrm{P}-\mathrm{O}$ bonding around $550 \mathrm{~cm}^{-1}$ and $1000 \mathrm{~cm}^{-1}$, and for $\mathrm{O}-\mathrm{H}$ bonding at $1650 \mathrm{~cm}^{-1}$ and around $3400 \mathrm{~cm}^{-1}$, respectively. From the results of XRD and FT-IR, the microspheres were found to con-
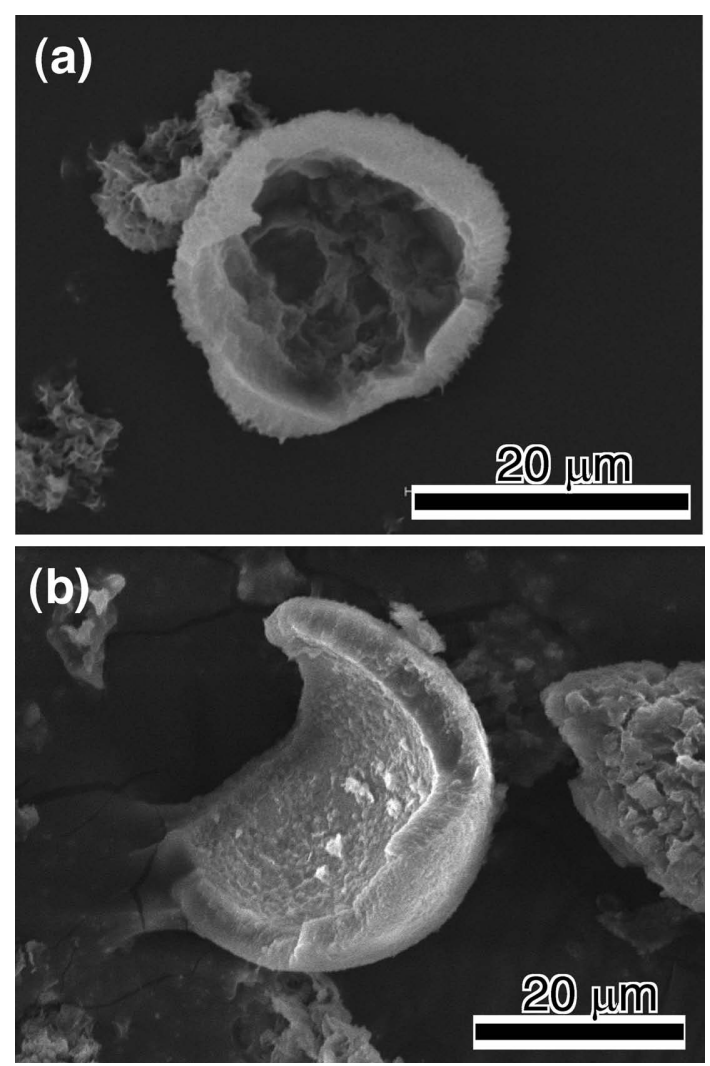

Fig. 6. SEM images of the cross-sectional views of the heat-treated samples for (a) 21.6 and (b) 432 ks of HAp precipitation.

sist of bone-like apatite.

Figure 6 shows SEM images of the cross-sectional views of the heat-treated, and deliberately broken samples for 21.6 and $432 \mathrm{ks}$ of HAp precipitation. The thickness of the shell for the sample soaked for $21.6 \mathrm{ks}$ is about $2-3 \mu \mathrm{m}$ and that for $432 \mathrm{ks}$ about 6-7 $\mu \mathrm{m}$. This means that the shell thickness of the HAp microspheres can be controlled by the duration of the enzymatic reaction.

\section{Conclusions}

HAp hollow microspheres a few tens micrometers in diameter were fabricated. The size of the HAp microspheres reflected the size of the template that was easily controlled in a range from 10 to $200 \mu \mathrm{m}$ during the preparation process of the template. The shell thickness of the HAp sphere was also regulated by the duration of the enzymatic reaction. These results and further examinations may confirm this material can be a candidate for a promising drug carrier.

\section{References}

1) P. van de Witte, H. Esselbrugge, A. M. P. Peters, P. J. Dijkstra, J. Feijen, R. J. J. Groenewegen, J. Smid, J. Olijslager, J. M. Schakenraad, M. J. D. Eenink and A. P. Sam, J. Control. Release, 24, 61-78 (1993).

2) C. V. Ragel and M. Vallet-Regi, J. Biomed. Mater. Res. Part A, 51, 424-429 (2000).

3) R. S. Byrne and P. B. Deasy, J. Microencapsul, 22, 423-437 (2005).

4) T. Akazawa, M. Kobayashi and K. Kodaira, Bull. Chem. Soc. Japan, 70, 2323-2329 (1997)

5) M. C. Sunny, P. Ramesh and K. K. Varma, J. Mater. Sci.: 
Mater. Med., 13, 623-632 (2002).

6) T.-J. Wu, H.-H. Huang, C.-W. Lan, C.-H. Lin, F.-Y. Hsu and Y.-J. Wang, Biomaterials, 25, 651-658 (2004).

7) K. Wei, Y. Wang, C. Lai, C. Ning, D. Wu, G. Wu, N. Zhao, X. Chen and J. Ye, Mater. Lett., 59, 220-225 (2005).

8) H.-W. Kim, B.-H. Yoon and H.-E. Kim, J. Mater. Sci.: Mater. Med., 16, 1105-1109 (2005).

9) C. C. Ribeiro, C. C. Barrias and M. A. Barbosa, J. Mater. Sci.: Mater. Med., 17, 455-463 (2006).

10) S. Teng, L. Chen, Y. Guo, J. Shi and J. Inorg, J. Inorg. Biochem., 101, 686-691 (2007).

11) X. Qui, Y. Han, X. Zhuang, X. Chen, Y. Li and X. Jin, J. Nanoparticle Res., 9, 901-908 (2007).

12) "An Introduction to Bioceramics," Ed. by L. L. Hench and J.
Wilson, World Scientific (1993).

13) T. S. Pradeesf, M. C. Sunny, H. K. Varma and P. Ramesh, Bull. Mater. Sci., 28, 383-390 (2005).

14) Q. Peng, L. Ming, C. X. Jiang, B. Feng, S. X. Qu and J. Weng, Key Eng. Mater., 309-311, 65-68 (2006).

15) Q. Wang, W. Huang, D. Wang, B. W. Darvel, D. E. Day and M. N. Rahman, J. Mater. Sci.: Mater. Med., 17, 641-646 (2006).

16) I. Kimura, T. Honma and R. E. Riman, J. Ceram. Soc. Japan, 115, 888-893 (2007).

17) H.-H. Lee, S.-J. Hong, C.-H. Kim, E.-C. Kim, J.-H. Jang, H.-I. Shin and H.-W. Kim, J. Mater. Sci.: Mater. Med., 19, 30293034 (2008). 